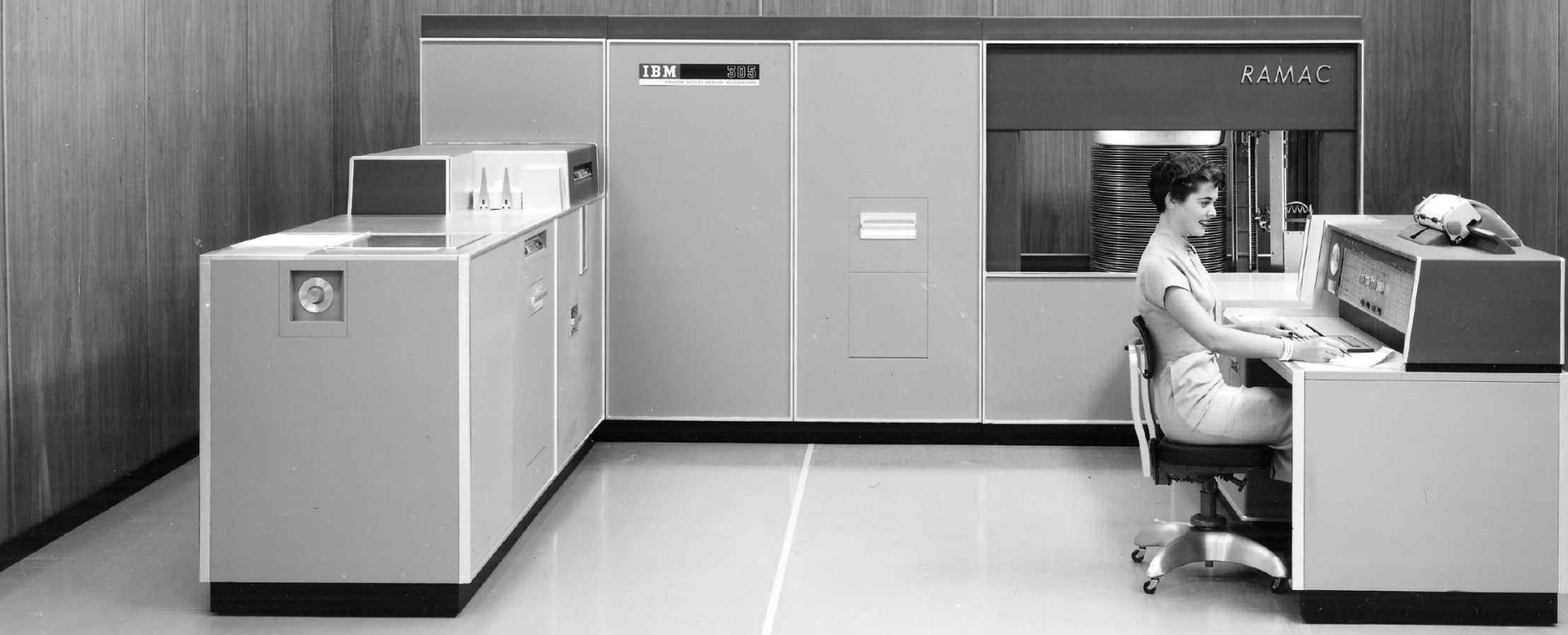

$\triangle$ FIC. 1: The IBM 305 RAMAC data processing computer, introduced in 1956, featured the world's first disk storage unit (IBM 350). Photo courtesy IBM.

\title{
CAN DATA BE STORED IN A SINGLE MAGNETIC ATOM?
}

》 DOI 10.1051/epn:2008404

Alexander F. Otte,

Leiden University - Current address: NIST, Gaithersburg (MD), USA n 1956, IBM introduced the 305 RAMAC data processing computer. The machine, shown in Fig. 1, was so big that it had to be delivered by cargo airplane. Its hard disk unit consisted of fifty 24" disks and had a total capacity of almost 5 megabytes. Compared to today's I-Pods and USB flash drives, which can store multiple gigabytes and are yet small enough to be accidentally left inside a pocket when doing the laundry, the kitchen-sized RAMAC makes a rather outdated and even comical impression. But that's really just an impression: technologically, the hard disk that is currently inside your laptop is almost exactly the same - only much smaller.

Unfortunately, the ongoing miniaturization of magnetic data storage as we know it (and inherently, the increase of the capacity) is soon coming to an end. That is because by reducing the size of the magnetic domains on the disk, we inadvertently also decrease the magnetic stability of these domains. Just how abrupt this ending will be is illustrated very well by the following example [1]. Take a magnetic domain that is just large enough to be stable, meaning that it can maintain its magnetization direction (i.e., the data) for, say, 10,000 years at room temperature. Now reduce the volume of this domain by only a factor of two: as a result of this seemingly small change, the magnetic decay time will suddenly drop by more than ten orders of magnitude to mere seconds! This merciless property of nature is known as the superparamagnetic limit.

Exactly at what domain size the superparamagnetic limit will take its toll depends on the type of magnetic material used. Or more precisely: it depends on how anisotropic that material is. For it is that property, magnetic anisotropy, that determines how well the domain's magnetic moment can tell one direction from the other. The more it prefers one specific axis to magnetize along, the less likely it is that the magnetic moment will swivel off in some random direction and thus erase the data it is supposed to represent. Therefore, control of the magnetic anisotropy of a material is the key towards stretching the limits of conventional magnetic data storage.

Nonetheless, the end of this process is near and will be here sooner than we would like. Therefore, rather than discussing incremental improvements, we will now consider a bold solution. Maybe even the boldest of all solutions: to make an individual magnetic atom hold a single bit of data. Seeing that the smallest bits currently available cover an area that can still host tens of thousands of atoms, this would be a stunning leap forward. Could it be done? Well, that depends on various things. First of all, it depends on what we mean by a 'magnetic atom'.

\section{Magnetism of an atom}

Magnetism is carried by the electrons on an atom. More specifically, it is carried by their intrinsic magnetic moment (spin), by their orbital angular momentum, or a by a combination of the two. In many cases the total amount of either quantity is zero. This is most notably the case when there are no partly filled 


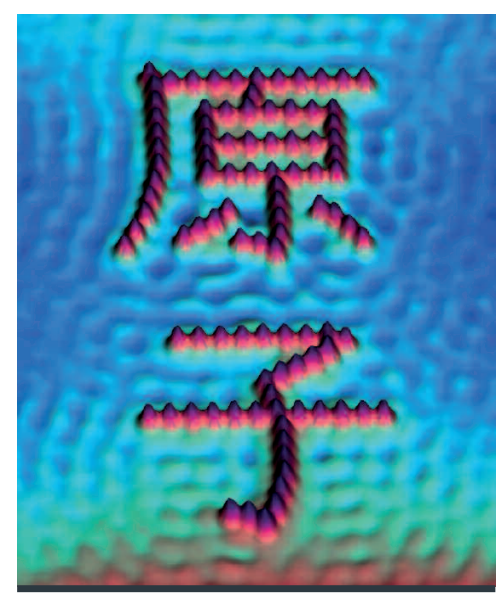

$\triangle$ FIG. 2: STM image of iron atoms on a $\mathrm{Cu}(111)$ surface. The atoms are arranged to form the Kanji characters for "atom". Image courtesy IBM. electronic shells on the atom, but happens also if a net amount of magnetic moment is 'consumed', for instance in forming a molecular bond with another atom. In each of these cases the atom is certainly not magnetic. But is the opposite also true? Is an atom that does have an uncompensated magnetic moment (such as an iron, cobalt, manganese or nickel atom) automatically magnetic? No, there is slightly more to it than just that.

Here we return to the anisotropy requirement mentioned above, which holds for individual atoms as well. A free atom suspended in space can never exhibit magnetism, regardless of its electronic configuration. If all directions are equivalent, it simply does not know which direction to magnetize in. So in order to make an atom magnetic, we need to place it in an anisotropic environment (for example, a solid state material). And that's exactly the idea of experiments recently published by Hirjibehedin et al. [2], which are described below: take a single atom with an uncompensated magnetic moment, place it onto an anisotropic surface and figure out (1) what direction it likes to magnetize in and (2) how badly it wants to point in that direction (i.e., how much effort it takes to make it point elsewhere).

\section{Seeing atoms and moving them around}

Maybe we should take a few steps back here. Because before we can even come close to putting an individual atom anywhere, we first need to be able to see it. This is where the Scanning Tunneling Microscope (STM) comes in, a device in which an atomically sharp needle (tip) scans along a surface in a manner strongly resembling a record player. While imaging, the STM tip does not contact the surface. Instead, it hangs a few tenths of a nanometer above the surface and maintains that separation by keeping the current of electrons tunneling through the vacuum gap between tip and surface constant. (For an introduction into the principles of STM, see ref. [3].) This way, when the tip scans over an atom that lies on top of the surface, its height temporarily increases such that the atom appears as a small bump.

But the STM can do much more than just imaging. Having seen the atoms, it can in some cases also move them around to any desired spot on the surface. In order to do so, the STM tip is lowered until it touches the atom, after which the atom can either be dragged along by the tip, or even be picked up and dropped off somewhere else. With this principle, first demonstrated in 1990 [4], increasingly complex structures (such as the
Kanji characters shown in Fig. 2) can be built on an incredibly small scale. This technology has its limitations though: in order for the atoms to stay put on their designated spot, temperature needs to be reduced to no more than a few Kelvins. Additionally, such atomic scale construction works should be performed in an ultra-clean vacuum environment as to prevent contamination by unwanted gas molecules.

\section{Spin excitation spectroscopy}

With this atom manipulation technique the first part of the single-atom-magnetism experiment is taken care of: we can pick up an atom and put it anywhere we want. Unfortunately, the second part is bound to be even more challenging: how do we figure out its magnetization state? Even for a macroscopic everyday magnet, finding the magnetization direction can be challenging. Most people would intuitively use the following method: first they would let the magnet relax into its preferred state (for instance stuck on top of another magnet) and then they would take it out of this state (rotate it, or pull it slightly off the magnet) while mentally recording how much effort each of these 'excitations' takes. After only a few such tests one can form a mental image of both the direction and the strength of the magnet's field. A very similar analysis can be performed on atomic spins, but there's one big difference: since atomic spins obey the laws of quantum mechanics, only a discrete set of welldefined excitations can be made to it. As it turns out, this property is highly useful for obtaining a detailed quantitative insight into the spin's behaviour.

The technique that was used to do this analysis is called Spin Excitation Spectroscopy. This technique consists of placing the STM tip over the magnetic atom of interest and slowly increasing the voltage applied between tip and surface. At low voltages little will happen: the atom's magnetic moment will point in its preferred (ground state) direction and tunneling electrons will peacefully pass through the atom on their way from the tip to the surface (Fig. 3a). But from a certain threshold voltage onward, the tunneling electrons injected into the
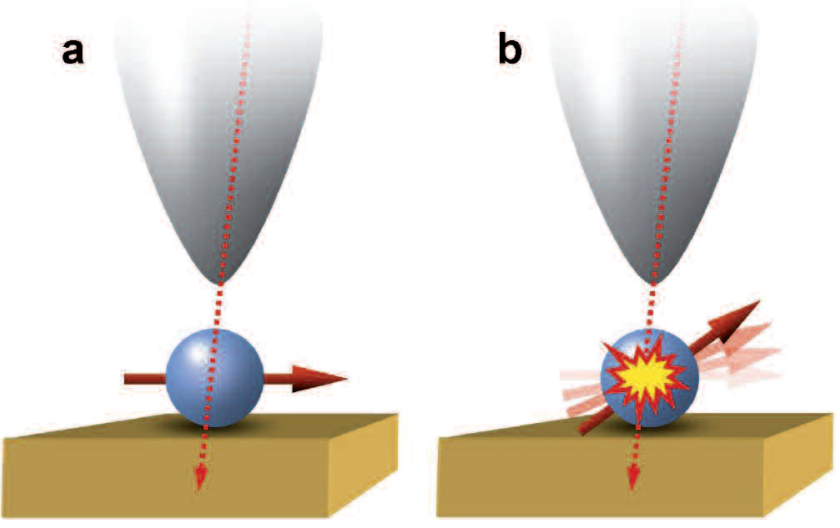

$\Delta$ FIG. 3: Above the threshold voltage electrons can tunnel through the magnetic atom either elastically (a) or inelastically (b). In the second case a spin excitation is made and the electron loses energy. Below the threshold voltage only elastic tunneling (a) is allowed. 
atom will have sufficient energy to perform an allowed excitation of the spin (thus losing some of their energy), and still have enough energy left to complete their journey into the surface. In this situation electrons can contribute to the tunneling current in two ways: either elastically by leaving the spin unchanged, or inelastically (Fig. 3b) by performing an excitation on the spin. As a consequence of this second tunneling possibility, the electrical conductance through the atom will show a sudden increase at the threshold voltage, telling us precisely the energy involved in the excitation.

In the experiments described in ref. [2], spin excitation spectroscopy measurements were performed on magnetic atoms lying on top of thin islands of copper-nitride $\left(\mathrm{Cu}_{2} \mathrm{~N}\right)$ that were grown on one of the facets of a copper crystal. These one atomlayer thick islands, which can be seen quite nicely in Fig. 4a, serve two purposes. First, they separate the atomic spins from the underlying metal, which is necessary for a clear definition of the spin's energy levels: by decoupling the localized spins from the free electrons in the metal the relaxation time of the spin increases as a result of which the levels become sharper.

But more importantly, the surface of these islands provides a strongly anisotropic environment that is ideal for the purpose of the experiment. Obviously, if an atom is placed onto any surface, the direction normal to the surface will always become unique. But if an atom is placed onto one of the copper atoms in the $\mathrm{Cu}_{2} \mathrm{~N}$ layer as shown in Fig. $4 \mathrm{~b}$, there is a clear distinction between the two in-plane directions as well. In one direction the atom is neighboured by two nitrogen atoms (the "N-direction"), while in the other direction it has two hollow sites as neighbours ("hollow direction"). This anisotropic situation was found to impose a distinct directionality on the spins of both manganese and iron atoms. For now, we will limit ourselves to the results obtained on an individual iron atom.

\section{Exciting results}

Figure 5 shows a measurement of the conductance (more precisely, the differential conductance, the derivative of the current to the voltage) through a single iron atom as a function of the voltage applied over the atom. The graph is almost symmetric, as it should be: the only difference between positive voltage and negative voltage is the direction of the flow of electrons, which should in principle not be relevant for performing spin excitations.

On either side the graph shows three distinct upward steps in conductance: one at less than $1 \mathrm{mV}$ (labelled red), one around $4 \mathrm{mV}$ (green) and one around $6 \mathrm{mV}$ (blue). Each one of these is a direct observation of an inelastic spin excitation performed by the tunneling electrons as discussed above. This is a fantastic result: it directly demonstrates that the iron atom has indeed found a preferential magnetization direction. Otherwise, if all directions would have the same energy, no finite-energy excitation would be possible and the conductance measurement would show a flat line.

But we are not quite there yet, because these steps do not tell us which direction this preferred direction is, or what kind

of excitations we are making. In order to find that out as well, strong magnetic fields were applied along the primary axes of the $\mathrm{Cu}_{2} \mathrm{~N}$ crystal surface. The idea of this method is that by seeing how the excitation energies change as a result of these fields, it might be possible to get an idea as to which step corresponds to which excitation of the spin. This idea turns out to work very well.

To start, let us see what happens to the iron atom's spin if a 5 Tesla magnetic field is applied along the $\mathrm{N}$ direction (Fig. 6a). For now we will focus on the 'blue' excitation (the one that used to be around $6 \mathrm{mV}$ ): it has gone up in energy and occurs now at least $1 \mathrm{mV}$ higher in voltage. This means that we still don't know what kind of excitation it is, but that whatever it is, it becomes harder to do it when the field points in the $\mathrm{N}$ direction. Next, we apply the same size magnetic field over the atom along the hollow direction (Fig. 6b): in contrast, now the blue step clearly goes down in energy. So whatever it is, it surely becomes easier to do with the field oriented like this.

With that we have found just enough clues to form ourselves a 'mental image' of the spin's orientation. An explanation that fits both observations is that the iron spin prefers to point in the $\mathrm{N}$ direction, and that it takes a tunneling voltage of about $6 \mathrm{mV}$ (corresponding to an electron energy of $6 \mathrm{meV}$ ) to turn it away from that direction in the plane of the surface. If we apply a magnetic field along that preferential direction, the spin gets pinned

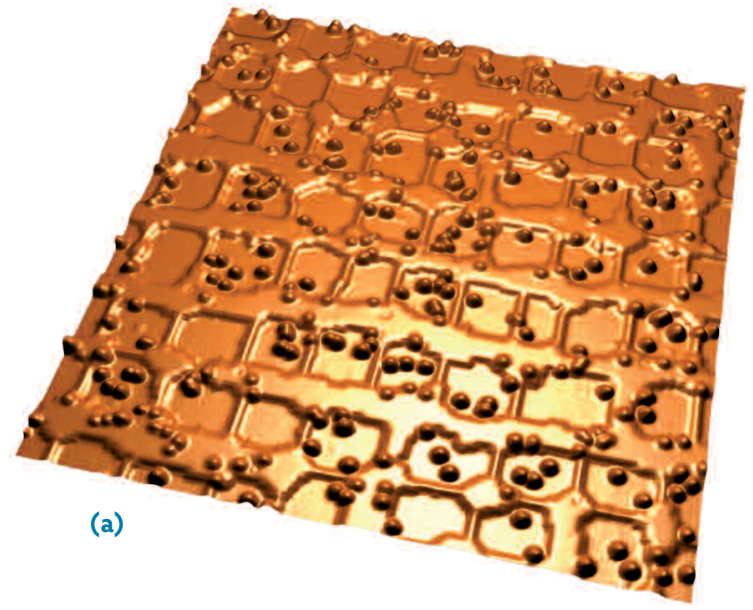

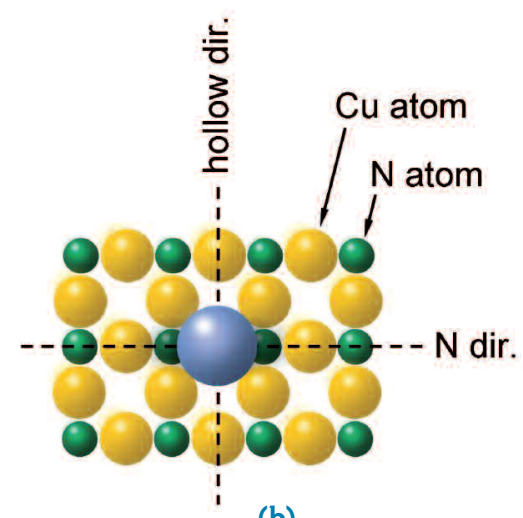

(b)
A FIG. 4: (a) STM image (approx. $50 \mathrm{~nm} \times 50 \mathrm{~nm}$ ) showing $\mathrm{Cu}_{2} \mathrm{~N}$ islands on top of a $\mathrm{Cu}(100)$ surface. The islands (one atom layer thick) appear as depressions rather than elevations as a result of the measurement technique. Magnetic atoms (in this image a mixture of iron, cobalt and manganese) are distributed randomly over the surface. (b) Crystalstructure of the $\mathrm{Cu}_{2} \mathrm{~N}$ surface. Labels indicate the hollow and N-directions for an iron atom bound atop a copper atom. 


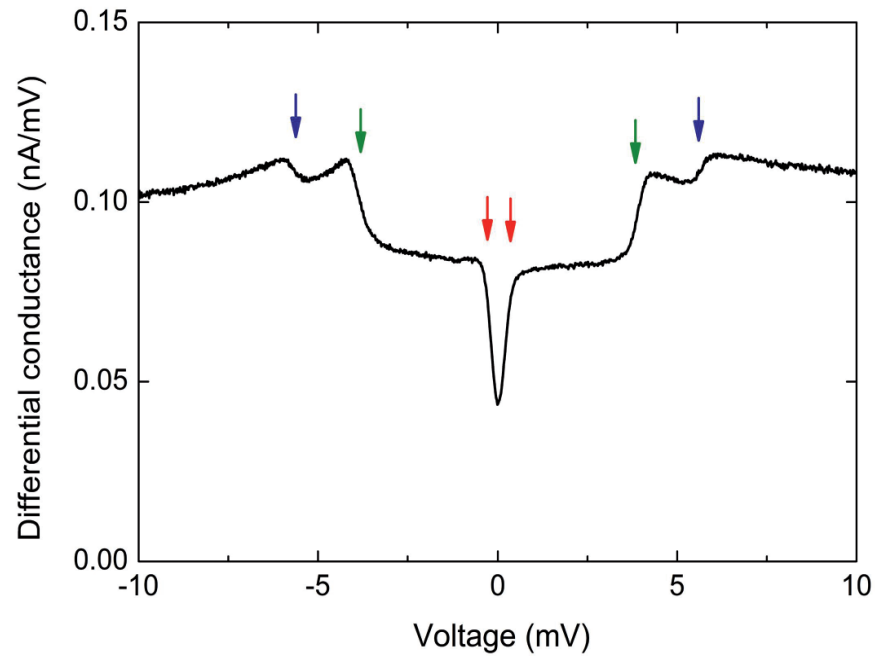

$\Delta$ FIG. 5: Spin Excitation Spectrum measured on a single iron atom. Steps occurring at finite energies indicate that the atom has magnetized.

down even stronger such that the rotation energy increases. But if we apply the magnetic field along the hollow direction, we 'help' the spin to rotate away: it all makes perfect sense.

A more involved analysis of the results has shown that this is indeed the case. The iron atom's magnetic moment behaves as a spin-2 system of which the ground state has full magnetization along the $\mathrm{N}$ direction (i.e. $\left|m_{\mathrm{N}}\right|=2$ ). The blue excitation puts the spin into an $\left|m_{\mathrm{N}}\right|=1$ state, with the majority of the remaining magnetization pointing in the hollow direction. Similarly, the green excitation (going up in energy in Fig. 6a while staying constant in Fig. 6b) brings the spin to a different $\left|m_{\mathrm{N}}\right|=1$ state: this time by rotating the spin towards the surface's normal axis rather than towards the hollow direction.

\section{Well? Can it hold data or not?}

Finally, the low-energy red transition corresponds to a full $180^{\circ}$ spin flip. This is the manipulation we would be most interested in from the viewpoint of magnetic data storage: it changes the bit from its ' 0 ' state $\left(m_{\mathrm{N}}=+2\right)$ to its ' 1 ' state $\left(m_{\mathrm{N}}=-2\right)$. At first sight this doesn't look too good: as seen in Fig. 5, only $0.2 \mathrm{meV}$ is needed to perform this flip, making the bit highly unstable. But that's just an illusion: due to the precise quantum mechanical nature of the situation, the ' 0 ' and ' 1 ' of the bit are not well defined in the absence of a magnetic field. Instead, the spin switches from one superposition of ' 0 ' and ' 1 ' to the other.

If, however, a magnetic field is applied along the $\mathrm{N}$ direction (Fig. 6a), this red transition quickly dies out. Already at 2 Tesla we can speak of clearly separated ' 0 ' and ' 1 ' states. In this situation, the only way to get from one side to the other is by making an actual rotation; either through the 'blue' path or through the 'green' one. And as we have seen above, these transitions take quite a bit of energy. Using these values one can calculate that an iron atom on $\mathrm{Cu}_{2} \mathrm{~N}$ could in principle remember its bit of information up to a few years, provided that it is cooled down below a temperature of approximately 2 Kelvin.
So the final answer is: yes, data can be stored in a single magnetic atom. But will atomic hard disks be available in your local store soon? Certainly not. Many technological obstacles still need to be overcome before this principle can be put to practice. First and foremost an atom/substrate combination should be found that provides an even higher energy barrier for the bit, such that it can be used at higher temperatures. It should be noted though, that the $2 \mathrm{~K}$ operating temperature mentioned above sounds more dramatic than it is. If a system were to be found that is stable above only $77 \mathrm{~K}$, the temperature of liquid nitrogen, applications in large-scale data centers could already become feasible.

A more serious objection, however, is that we now only know that an atom can hold information. How the information should be written onto it and how it can be retrieved are different issues altogether that require independent investigation. Another complication comes with the fabrication of atomic-scale storage media: while the STM is useful for arranging atoms one at a time, no device is to be expected any time soon that can do the same thing on an industrial scale. At least, probably not soon enough to beat the superparamagnetic limit.

\section{About the author}

Alexander F. Otte currently works as a Postdoc at the Center for Nanoscale Science and Technology at NIST in Maryland. In early 2008 he received his $\mathrm{PhD}$ from Leiden University. His thesis work consisted of the development of a ${ }^{3} \mathrm{He}$ STM, as well as research on Spin Excitation Spectroscopy in collaboration with the IBM Almaden Research Center.

\section{References}

[1] R.L. White, J. Mag. Mag. Mat., 209 (2000) 1

[2] C.F. Hirjibehedin et al., Science, 317 (2007) 1199

[3] H.-J. Güntherodt and R. Wiesendanger, Scanning Tunneling Microscopy I (Springer Verlag, Berlin/Heidelberg, 1992)

[4] D.M. Eigler and E.K. Schweizer, Nature, 344 (1990) 524
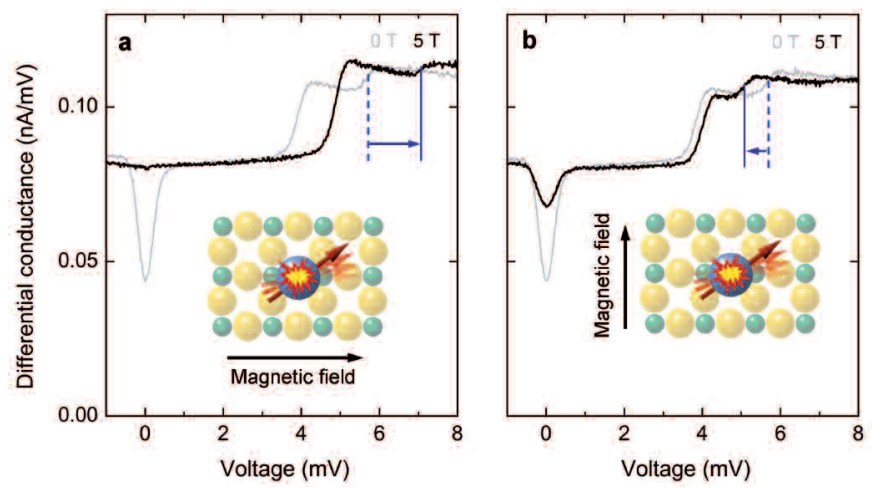

$\Delta$ FIG. 6: When magnetic fields up to 7 Tesla are applied along the Ndirection (a) or hollow direction (b), the observed excitation energies evolve differently. Specifically the 'blue' excitation (as defined in Fig. 5) goes up in energy in one case and down in the other. The insets show schematically what is happening: in (b) the magnetic field assists the excitation while in (a) it makes the excitation more difficult. 